\title{
Peptidoglycan Remodeling by the Coordinated Action of Multispecific Enzymes
}

\author{
Laura Alvarez, Akbar Espaillat, Juan A. Hermoso, ${ }^{2}$ Miguel A. de Pedro, ${ }^{3}$ and Felipe Cava ${ }^{1}$
}

The peptidoglycan (PG) cell wall constitutes the main defense barrier of bacteria against environmental insults and acts as communication interface. The biochemistry of this macromolecule has been well characterized throughout the years but recent discoveries have unveiled its chemical plasticity under environmental stresses. Non-canonical D-amino acids (NCDAA) are produced and released to the extracellular media by diverse bacteria. Such molecules govern cell wall adaptation to challenging environments through their incorporation into the polymer, a widespread capability among bacteria that reveals the inherent catalytic plasticity of the enzymes involved in the cell wall metabolism. Here, we analyze the recent structural and biochemical characterization of Bsr, a new family of broad spectrum racemases able to generate a wide range of NCDAA. We also discuss the necessity of a coordinated action of PG multispecific enzymes to generate adequate levels of modification in the murein sacculus. Finally, we also highlight how this catalytic plasticity of NCDAAincorporating enzymes has allowed the development of new revolutionary methodologies for the study of PG modes of growth and in vivo dynamics.

\section{Catalytic Plasticity}

D URING THE LAST DECADES, the classical idea of "one enzyme-one substrate-one reaction" has been progressively broadened by a number of reports illustrating that a great variety of enzymes can accept multiple substrates, and even mediate additional-maybe secondary-catalytic reactions (see reviews ${ }^{10,39}$ ). Catalytic plasticity comprises both promiscuous and multispecific activities. The term catalytic promiscuity has been coined as the ability of an enzyme to catalyze multiple reactions ${ }^{12,42}$ (i.e., cleaving different bonds), whereas the concept of substrate specificity refers only to the ability of enzymes to transform either one (monospecific enzymes) or more (multispecific enzymes) substrates.

The study of catalytic promiscuity can provide insights into the evolution of the enzymes. On the one hand, catalytic promiscuity could be considered a vestige from ancestral forms whose active sites had little selectivity; on the other hand, acquisition of an advantageous additional activity promoted by gene duplications and accumulation of mutations can give place to emerging enzymatic families, not necessarily at the expenses of the native activity. Several studies on diverse superfamilies of enzymes have explored the evolutionary mean- ing of promiscuity and multispecificity, ${ }^{4,28,60}$ but results are far from being conclusive.

The catalytic plasticity of an enzyme is encrypted in its structure. Enzymes improve the catalytic efficiency of a process by adopting different conformations ${ }^{41}$ or reduce the energetic requirements of a reaction by simply displaying flexibility at their active site. ${ }^{34,37,61}$ This can be readily illustrated by the conformational changes that occur, for example, in ligand recognition, allosteric regulation, protein-protein interactions, signal transduction, or the assembly of multiprotein complexes. ${ }^{26,30,64,75}$ Moreover, structural flexibility is also an important evolutionary trait in the adaptation of proteins to different environments- such as extreme conditions. ${ }^{16}$ Additionally, since enzymes catalyze a wide variety of biological processes, their structure flexibility has ultimately a great impact in their biological functions. Enzymatic structural flexibility determines the degree of substrate/effector accessibility to its active site, binding efficiencies and reaction kinetics (reviewed in Kokkinidis et $a l^{45}$ ). The importance of conformational flexibility for catalytic efficiency has promoted the development of new methodologies such as molecular dynamics and nuclear magnetic resonance that permit investigation of protein mobility at time scales of femtoseconds to seconds. ${ }^{49,54}$

\footnotetext{
${ }^{1}$ Laboratory for Molecular Infection Medicine Sweden, Department of Molecular Biology, Umeå Centre for Microbial Research, Umeå University, Umeå, Sweden.

${ }^{2}$ Department of Crystallography and Structural Biology, Instituto de Química-Física "Rocasolano," CSIC, Madrid, Spain.

${ }^{3}$ Centro de Biología Molecular "Severo Ochoa," Universidad Autónoma de Madrid-Consejo Superior de Investigaciones Científicas, Madrid, Spain.
} 
A more comprehensive understanding on catalytic plasticity will need multidisciplinary approaches combining phylogenetic analysis, functional considerations of the genetic context, in silico substrate docking simulations, and fine biochemical characterization. Deciphering the molecular basis of catalytic plasticity will shed light on the versatility of the active and accessory sites ${ }^{4,20}$ and therefore will boost the discovery of novel metabolic and regulatory processes in living organisms and their potential use in biotechnology.

Here, we review current research on the coordinated action of multispecific enzymes that confer adaptive plasticity to the murein sacculus. We make particular emphasis on the biochemical and structural aspects of these enzymes as the driving force that underscores such cell wall variability.

\section{Plasticity in the Cell Wall}

The peptidoglycan (PG) layer is an essential cell wall envelope component present in most bacteria that confers the characteristic shape and size, protection against environmental challenges and plays a key role in growth and division. ${ }^{84,86}$ This polymer is composed of glycan strands cross-linked by short peptides. ${ }^{6}$ The structure of the basic unit is highly conserved among bacterial species. Glycan strands are composed of $N$-acetylglucosamine (GlcNAc) and $N$-acetylmuramic acid (MurNAc) linked by $\beta 1 \rightarrow 4$ bonds. The peptide chains somewhat vary among bacterial species, but normally include the D-amino acids D-Ala and D-Glu (or its amidated form, D-Gln). PG precursors are synthesized by the sequential action of several cytoplasmic ligases, ${ }^{5}$ linked to GlcNAc and finally flipped to periplasmic space. ${ }^{14,79}$ There, bifunctional penicillin-binding proteins (PBPs) displaying PG glycosyltransferase (GT) and transpeptidase (TP) activities polymerize the glycan chains and cross-link muropeptides to the pre-existing PG network, either directly or by a short peptide bridge. ${ }^{15,71,78}$ The PG sacculus is a highly dynamic macromolecule, which is subjected to constant alterations in response to changing environmental conditions (PG plasticity). ${ }^{39}$

The plasticity of the PG is largely the consequence of modifications taking place concomitantly with, or at different stages, after its synthesis. These modifications can be considered as "editing" or "remodeling" processes that affect both the glycan strand and the peptide stems and require the action of specific enzymes (i.e., acetylases, deacetylases, monooxygenases, TPs, endopeptidases, carboxypeptidases etc.). ${ }^{81,83,84}$ Many studies underscore the importance of PG plasticity in bacterial growth and survival. ${ }^{17,39,77,81}$ Indeed, PG properties change constantly as the cell population ages. In Escherichia coli, the chains are shortened during stationary phase while cross-linkage between muropeptides (specially mDAP-mDAP bridges) and binding of Braun's lipoprotein (Lpp) are increased. ${ }^{29}$ These changes occur as a consequence of the maturation of the sacculus and a diminished incorporation of new units to the PG structure. Another example of PG rearrangements elicited by long-term cultivation is the morphological transition to persistent coccoid forms developed by the human gastric pathogen Helicobacter pylori. ${ }^{1}$

PG editing is also a mechanism devised by pathogenic bacteria like Streptococcus pneumoniae, Listeria monocytogenes, or Bacillus anthracis to evade the immune system. $10,63,82,92$ These modifications include deacetylation or acetylation in the glycan strands or increased levels of tei- choic acids or cross-linking, which ultimately prevent degradation by lysozyme or related hydrolytic enzymes produced by the host. ${ }^{7}$ For instance, Bacillus cereus strains exhibiting a high percentage of non-acetylated glycan strands show resistance to lysozyme. ${ }^{3,92}$ In the case of $O$-acetylation, it has been shown that most muramidases, except for the N,Odiacetylmuramidase from Chalaropsis ${ }^{36}$ or mutanolysin from Streptomyces globisporus, ${ }^{33}$ have reduced or no activity against modified PG. Another example is the pneumococcal teichoic acid phosphorylcholine esterase (Pce [or CbpE]) that is capable of removing a limited number of phosphorylcholine (PC) residues from pneumococcal cell walls. The crystal structure of $\mathrm{Pce}^{38}$ revealed that it would be involved in specifically releasing only those PC terminal residues relevant for cell-cell interactions. Selectively modifying the distribution of the PC moieties on the bacterial surface by Pce impairs recognition by host immune components, such as human C-reactive protein. Therefore, cell wall editing by PC removal is a mechanism for pneumococci to escape the host immune system.

\section{Production of Non-Canonical D-Amino Acids: BsrV}

D-alanine and D-glutamic acid are basic components of the bacterial cell wall, ${ }^{27,35,65}$ present in the stem peptides of the PG network. ${ }^{84}$ This has been hypothesized to be a resistance mechanism to most known proteases as an adaptive mechanism to preserve an essential cellular structure like the bacterial envelope. Other D-amino acids are usually present at the terminal position of the peptide stem in some bacteria, like D-serine $22,31,67,73$ or D-aspartic acid, ${ }^{6,80}$ to confer resistance against vancomycin and similar antibiotics.

Recent studies indicate that D-amino acids may have many unknown roles related with PG plasticity yet to be elucidated in the microbiology field. In 2009, Lam et al. described the production and release to the extracellular media of a set of D-amino acids by the pathogen Vibrio cholerae. ${ }^{48}$ Even more interestingly, these molecules can be incorporated to the cell wall, as constituents of the peptide stems of muropeptides by different pathways, ${ }^{17,18,39}$ thus editing the canonical PG composition and structure and constituting a novel mechanism of cell wall adaptation to environmental stresses (i.e., starvation and stationary phase). This investigation demonstrated that not only $V$. cholerae but also many other microorganisms, taxonomically unrelated, produce a wide set of non-canonical D-amino acids (NCDAA), which are released to the extracellular media. ${ }^{48}$

The metabolic investment in production of NCDAA reflects their important physiological role and revives old literature that pointed their toxic effects on the cell wall of different bacteria. ${ }^{11,25,32,44,50,74,76,88}$ Indeed, NCDAA can act as a new engine of $\mathrm{PG}$ diversification and interspecies communication in poly-microbial communities, since non-producer organisms can nonetheless be influenced by NCDAA either with beneficial or antagonistic consequences.

There have been reported additional roles for NCDAA other than cell wall modulation. For instance, Bacillus subtilis produces a different set of NCDAA compared to $V$. cholerae (DLeu, D-Met, D-Trp, and D-Tyr), and it uses them as a biofilm dispersal strategy. Although the mechanism was initially believed to affect TasA-mediated cell-matrix connections, ${ }^{43}$ recent data points toward protein synthesis interference as the actual mechanism leading to biofilm disassembly. ${ }^{46,53} \mathrm{D}-\mathrm{Ser}$ is 
a signal in colonization and its level regulates virulence gene expression in uropathogenic E. coli. ${ }^{2}$ In Myxococcus xanthus, many $\mathrm{D}$-amino acids mimic $\beta$-lactam antibiotics triggering induction of the $\beta$-lactamase, which induces sporulation as a mechanism to resist cell wall damages and promote cell survival. $^{62}$

Interconversion of L- to D-amino acid occurs mainly by the action of racemases that change the chiral $\alpha$-carbon atom in amino acids through a deprotonation/reprotonation mechanism. There are two major mechanistic groups of amino acid racemases distinguished by their dependence (PLP-dependent) or independence (PLP-independent) from the cofactor pyridoxal5-phosphate (PLP). The first racemase described in 1951 was a bacterial alanine racemase (Alr). ${ }^{87}$ Since then, a considerable number of similar enzymes have been reported in bacteria, archaea, and eukaryotes (reviewed in Yoshimura and Esak $^{91}$ and Conti et al. ${ }^{19}$ ).

Most racemases described exhibit high substrate specificity. However, a new family of racemases with broader substrate specificity (Bsr, for broad spectrum racemases) has been recently reported. ${ }^{24}$ The broad spectrum racemase from $V$. cholerae $(\mathrm{BsrV})$ is the paradigm of these multispecific enzymes. Espaillat et al. have characterized the 3D structure of this family and through extensive mutational, biochemical, and bioinformatic analyses they have revealed the unique molecular determinants (absent in monospecific racemases) that define the structural bases for BsrV multispecific activity. ${ }^{24}$ This molecular signature has permitted in silico identification of more Bsr in bacteria and confirm those previously reported. $40,56,69,89,90$

\section{Structural determinants of multispecificity in BsrV}

Surprisingly, Bsrs show a great similarity to the substrate stringent Alrs. In fact, the miss-annotation of BsrV as a second Alr (AlrV) in V. cholerae's genome has probably hampered its identification before. Both BsrV and AlrV are PLP-dependent racemases that dimerize to form the active sites. Unlike AlrV, which is cytoplasmic and racemizes only alanine and serine, BsrV is a periplasmic racemase that can use 10 (Ala, Leu, Met, Ser, Cys, Gln, Asn, His, Lys, and Arg) of the 19 natural chiral amino acids. ${ }^{24}$

The study of BsrV structure and its comparison to E. coli Alr reveals important structural features that account for its multispecificity. As shown in Figure 1, BsrV presents a
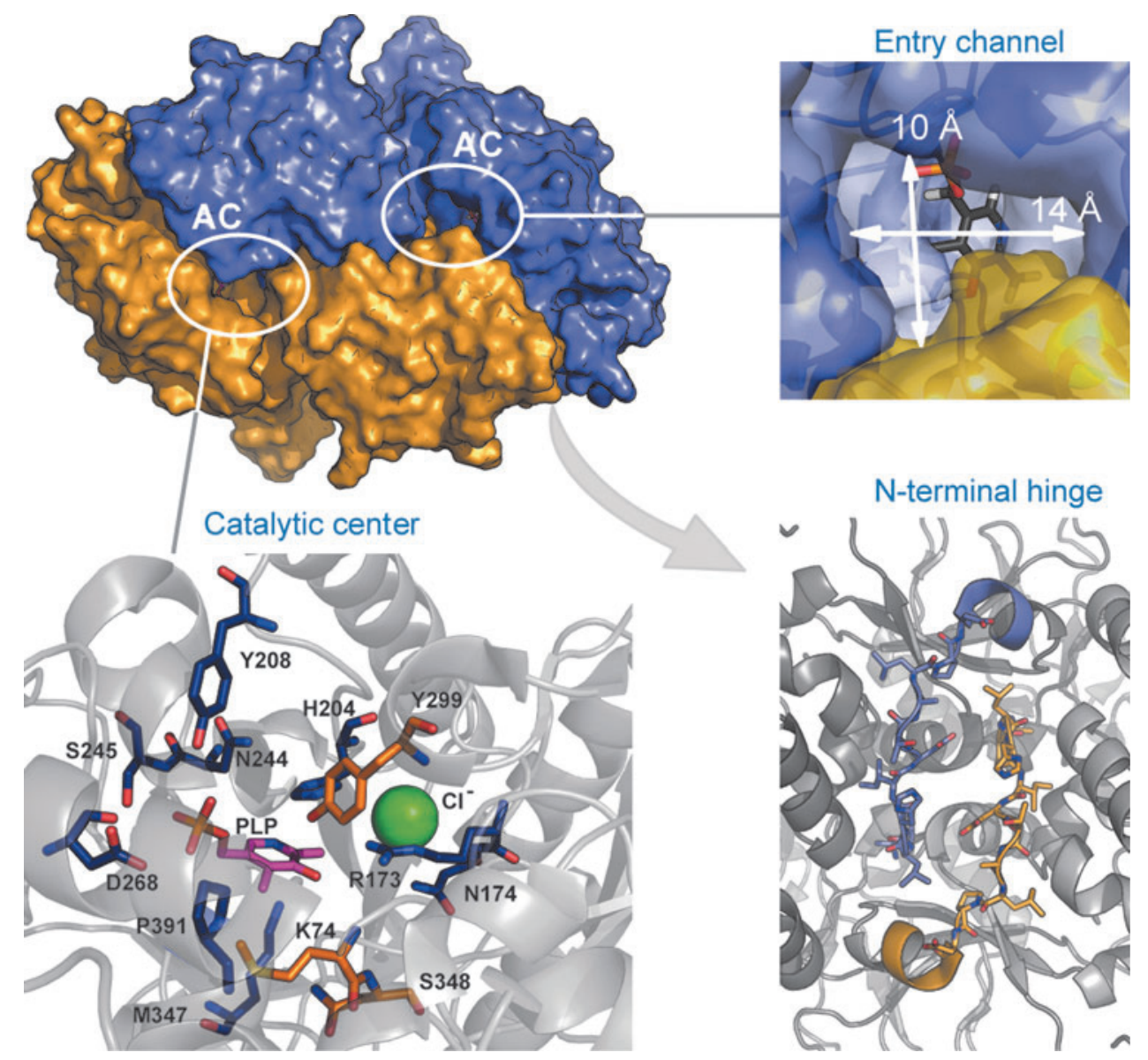

FIG. 1. Crystal structure of the broad spectrum racemase (Bsr) of Vibrio cholerae. BsrV (PDB: 4BEU ${ }^{24}$ ) is a homodimer with three special features that define its multispecificity: (i) it presents a wide entry channel that allows allocation of a diverse set of non- $\beta$-branching aliphatic and positively charged amino acids; (ii) the N-terminal extension of both monomers establishes polar interactions that improve the stability of the dimer and provokes a change in the disposition of the entry channels versus that observed for alanine racemase (Alr); (iii) and the catalytic machinery differs from that of Alrs as it presents a $\mathrm{Cl}^{-}$ion (in green) instead of the typical N-carboxylated lysine, and several residues involved in pyridoxal-5-phosphate (PLP) coordination and substrate stabilization that constitute a molecular signature specific of this type of enzyme. AC, active center. 
rather wide entry channel that allows the entrance of a broader variety of substrates. The amino acid composition of the entry channel also accounts for substrate selectivity; negatively charged amino acids are excluded due to an accumulation of negative charges at the catalytic entry site. The tunnel that connects the entry and the active site is also wider in BsrV. Interestingly, BsrV presents an $\mathrm{N}$-terminus extension that confers stability to the dimerization by forming a hinge-like structure between monomers. This conformational rearrangement in BsrV N-terminus elicits a shifted disposition of the subunits compared to that reported for Alrs and, thus, an important difference in the location and orientation of the active sites of both enzymes.

The active sites also differ in their size and disposition. Replacement of the Tyr residue that coordinates the PLP cofactor in Alr by a Pro residue enlarges the active site in BsrV, allowing the stabilization of larger substrates. Unexpectedly, the substrate binding is stabilized in BsrV by a tetracoordinated $\mathrm{Cl}^{-}$ion in contraposition to the $\mathrm{N}$-carboxylated Lys and Arg residues that perform this task in most Alrs. ${ }^{85}$ In this case, the conservation of the catalytic Lys and Tyr residues in both Alr and Bsr indicates that the reaction mechanism is similar in both families of racemases.

\section{Prediction of new families of racemases}

Bioinformatic analysis of the Bsr and Alr families shows that only Gram-negative bacteria present this type of Bsr. ${ }^{24}$ The use of multi-sequence alignment algorithms has allowed the identification of the molecular footprints and determinants of Bsr multispecificity. Since some Grampositive organisms like $B$. subtilis have also been reported to produce a different set of NCDAA, ${ }^{46,48}$ additional families of broad-spectrum enzymes remain to be identified. The finding of this previously unnoticed family of Bsr highlights the necessity to keep exploring the existence of novel multispecific activities in nature. A combination of methodologies like computational predictions, crystallography, structure characterization, and biochemical assays will allow the discovery of such families of plastic enzymes of great potential for both the biotechnological and pharmaceutical fields. $^{24}$

\section{Coordinating NCDAA Production with the PG Synthetic and Remodeling Pathways}

Lam et al. demonstrated that NCDAA produced by $V$. cholerae (i.e., D-Met) were incorporated into the cell wall. This observation gave rise to a new family of modified muropeptides (NCDAA-muropeptides) ${ }^{48}$ and renovated the traditional concept of PG being a conserved and static entity. It is worth mentioning that the capacity to incorporate NCDAA is widespread in bacteria. In fact, non-producer organisms can nonetheless incorporate NCDAA from the media in mixed microbial communities leading to phenomena of interspecies communication. NCDAA-muropeptides present DAA variants, different from D-Ala, at position 4 or 5 of the peptide stems. Interestingly, incorporation at either site requires involvement of different enzymes and pathways able to accommodate the atypical DAA (Fig. 2). Furthermore, this means that these NCDAA-muropeptides can act both as substrates and/or regulatory effectors of the enzymes that synthetize and remodel the murein sacculus. The degree of permissiveness of PBPs to NCDAA-muropeptides was investigated by Lebar et al. Modified lipid II precursors in which mDAP had been chemically replaced by L-Lys were recognized by PBPs in vitro and polymerization was possible, but no transpeptidation, ${ }^{51}$ revealing certain degree of enzymatic flexibility to changes in the composition of the peptide.

Incorporation at the fourth position of the peptide stem of muropeptides is catalyzed by LD-transpeptidases (Ldts). Ldts are enzymes able to transfer a peptide bond linking an L- and a D-amino acid to a nucleophilic acceptor. This family of enzymes catalyze two major reactions in PG metabolism, one involved in dimer synthesis through the formation of mDAPmDAP peptide bridges, ${ }^{57,59}$ and another responsible for covalent attachment of Braun's Lpp to PG. ${ }^{58}$ Interestingly, Cava et al. demonstrated that the physiological production of NCDAA by BsrV was incorporated in PG-tetrapeptides (muro4) by Ldts. ${ }^{18}$ In fact, they demonstrate that in $V$. cholerae both Ldts account for the presence of muropeptides with D-Met in their fourth position. Interestingly, in vitro assays with purified LdtA showed that this enzyme can exchange the C-terminal D-Ala by virtually any NCDAA. As expected, the enzyme was unable to incorporate the L-enantiomers, showing a strict stereo-selectivity. These observations were consistent with previous studies on $\mathrm{Ldt}_{\mathrm{fm}}$ from Enterococcus faecium, which also recognizes several D-amino acids but no L-Met. ${ }^{59}$

The crystal structure of the C-terminal domain of YkuD from $B$. subtilis and the catalytic domain of $\mathrm{Ldt}_{\mathrm{fm}}$ of E. faecium were described in $2006^{8,9}$ and the structures of Ldts Mt1 and Mt2 from Mycobacterium tuberculosis have been reported recently. ${ }^{13,21,23,43}$ In this work, a reaction mechanism was proposed in which both the acyl-acceptor and the acyl-donor substrates enter the catalytic site through the same entry. All enzymes so far described present similar two-domain architectures and highly conserved catalytic domains, being only slightly distinct at their N-terminal domain. Unfortunately, the molecular determinants of Ldts multispecificity have not been described yet.

Incorporation of NCDAA at the fifth position can be explained by two distinct pathways: (i) by an amino acid exchange mediated by the action of DD-acyltransferases (carboxypeptidases, TPs) or (ii) by synthesis of modified precursors in the cytoplasmic de novo synthetic pathway. ${ }^{18,39}$ The first case requires the presence of pentapeptides as substrate. However, $V$. cholerae and many other bacteria exhibit undetectable amounts of pentapeptides in their PG because of the concerted action between TPs and carboxypeptidases. Removal of the terminal residues of the muropeptides is carried out by two types of carboxypeptidases: the DD-type (DD-CP) remove the amino acids on fifth position, while LD-carboxypeptidases (LD-CP) eliminate the residues in fourth position. However, the latter are cytoplasmic enzymes involved in muropeptide recycling, ${ }^{66}$ with the exception of the periplasmic LD-CP of Proteus vulgaris. ${ }^{70}$ The substrate specificity of the E. coli LD-CP has been well characterized. ${ }^{52}$ The enzyme is specific for tetrapeptides and discriminates against pentapeptides, but is permissive with regard to the attached sugar moieties.

The second mechanism requires that cytoplasmic ligases Ddl and MurF allow incorporation of alternative D-amino acids to the peptide stem. In vitro studies on precursor 


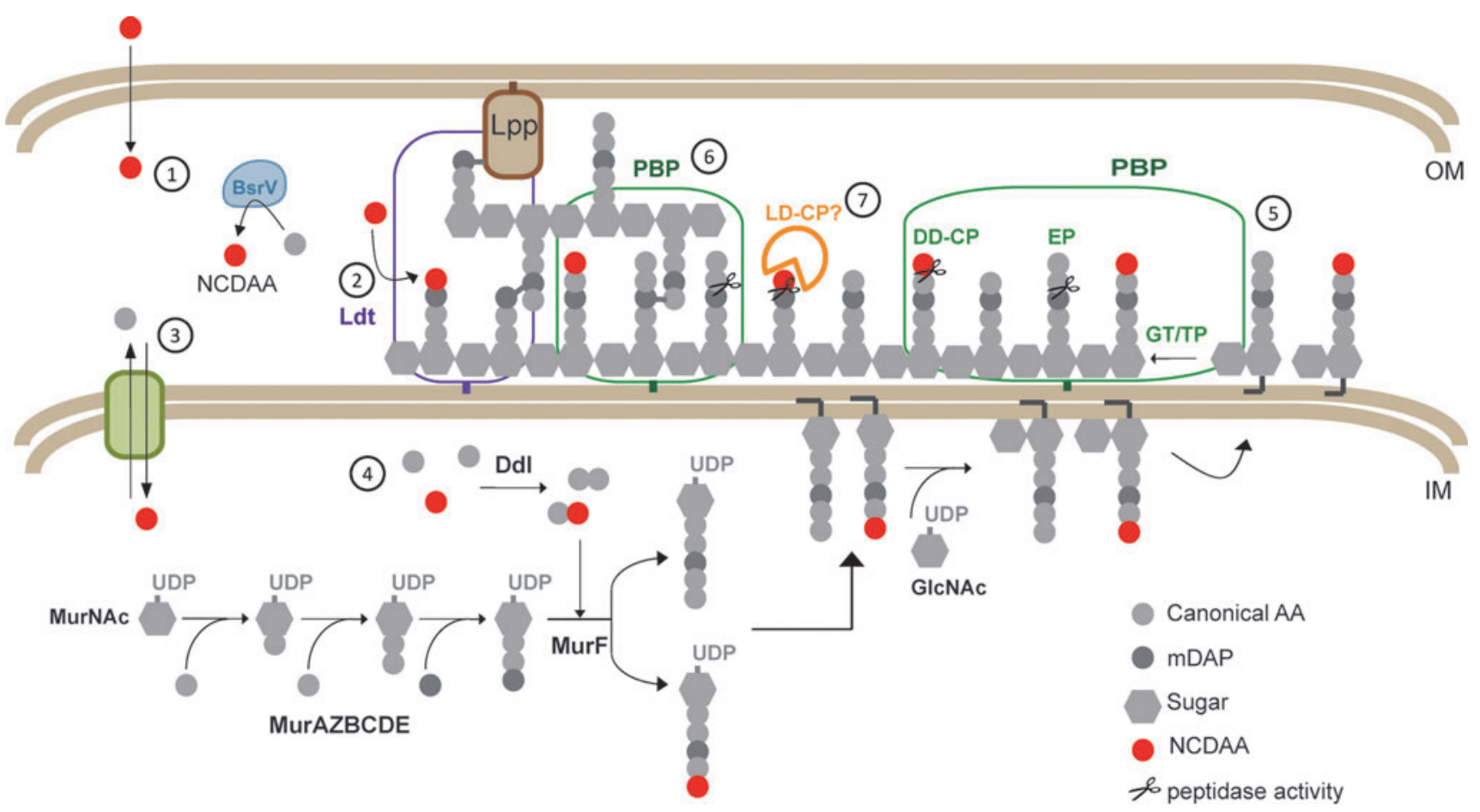

FIG. 2. Pathways of non-canonical D-amino acids (NCDAA) incorporation in the cell wall of $V$. cholerae. (1) NCDAA can be acquired from the extracellular media or they can be produced extra-cytoplasmically by BsrV. (2) They can be incorporated into the fourth position of the peptide moiety by the action of LD-transpeptidases (Ldts). (3) ABC transporters provide LAA to the periplasmic BsrV and facilitate entrance of NCDAA, which can be used in the synthetic pathway of the peptidoglycan (PG) precursors. (4) Promiscuous Ddl and MurF ligases allow generation of NCDAA-modified Lipid II precursors. $(5,6)$ The synthetic penicillin-binding proteins (PBPs) polymerize and cross-link the PG through their glycosyltransferase (GT) and transpeptidase (TP) activities. Accumulation of modified pentapeptides depends on their DD-carboxypeptidase (DD-CP) or endopeptidase activities (EP) and their affinity to modified substrates. (7) Putative periplasmic LD-carboxypeptidase (LD-CP) activity could also account for the hydrolysis of modified tetrapeptides. OM, outer membrane; IM, inner membrane.

synthesis in presence of D-Ala or D-Met confirmed that $V$. cholerae's Ddl and MurF are promiscuous enough to catalyze the generation of UDP-(D-Met)-pentapeptides. ${ }^{18} \mathrm{~A}$ similar mechanism of PG plasticity at the fifth position of the muropeptides accounts for resistance to vancomycin in Enterococcus and Staphylococcus aureus. Vancomycin specifically binds to the D-Ala-D-Ala moiety in muropeptides thus interfering with PG synthesis. ${ }^{22,67}$ Vancomycin-resistant organisms have developed a set of enzymes that produce a modified precursor in which a D-Ser substitutes the terminal residue of the peptide stem making it impervious to the antibiotic. This system requires the coordination of several enzymes: a serine racemase that produces the D-Ser, a flexible Ddl ligase able to recognize and catalyze the synthesis of D-Ala-D-Ser, and a MurF able to recognize the modified dipeptide and bind it to the MurNAc-tripeptide precursor.

The ability of so many different species to incorporate D-amino acids is an indication of a much larger degree of plasticity of the PG biosynthetic pathway than originally expected. Regardless of the mechanism that bacteria have chosen to produce edited PG-pentapeptides, it is likely that incorporation of NCDAA has a great impact in the physiological architecture of the bacterial PG in nature given that PG-ligases and PBPs are prevalent activities in virtually all bacteria and that NCDAA are present at high concentration in the environment.

\section{Exploitation of PG Enzymatic Plasticity as Source for Research Tools}

Promiscuous incorporation of NCDAA in the PG has led to the development of new methods for the study of PG dynamics. ${ }^{47,72}$

In $B$. subtilis incorporation of fluorescent derivatives of DAA (FDAA) occurs only at fifth position. Fluorescentmuro5 are accumulated to a higher extent in DD-carboxypeptidase mutants, which indicates that these hydrolytic enzymes might be key check points in the control of modified muropeptides levels at the sites of active PG-synthesis. In contraposition, incorporation of FDAA in E. coli and Agrobacterium tumefaciens occurs at fourth position. Given the absence of so-far-known extra-cytoplasmic LD-CP, it seems that the only activity modulating the levels of NCDAAmuropeptides can be Ldts themselves, either by performing reverse reactions whereby an NCDAA-tetrapeptide is reconverted into a canonical one in the presence of $\mathrm{D}$-alanine as acceptor (NCDAA-muro4+D-Ala > muro4 + NCDAA); or by LD-cross-linking activity whereby the donor NCDAAmuro4 releases the NCDAA concomitantly with the formation of a LD-dimer muropeptide (NCDAA-M4+M4> D34 + NCDAA). In any case, it is needed that all enzymes act coordinately to allow proper cell wall integrity during cell growth and division. Nonetheless, the possibility exists that 
several mechanisms of incorporation concur at the same time and space to ensure a correct balance.

Alternative to FDAA, bioorthogonal chemistry has also provided a new method to track PG dynamics in diverse bacteria based on the same principle of PG plastic incorporation of amino acid derivatives. ${ }^{72}$ As anticipated, the use of visualization tools to deepen into the PG metabolism is becoming an extraordinary useful tool to explore fundamental questions in cell wall biology. As an example, Liechti et al. have recently used these PG-labeling strategies to demonstrate the existence of PG in chlamydial species, ${ }^{55}$ something that was unsuccessfully attempted in the past by conventional PG analytical methods.

\section{Conclusions}

Catalytic plasticity is a property of certain enzymes that permit the generation of diverse reaction products. Although catalytic plasticity is rarely linked to enzymatic efficiency (who focuses upon everything has, in fact, no focus), it has probably evolved to provide a selective advantage for the organism to adapt to changing environments.

PG modulation by NCDAA represents a perfect example of a process driven by a set multispecific enzymes whose activities need to be coordinated for a common goal: adapt bacterial PG to stationary growth phase conditions. Under stationary phase, and likely other RpoS regulated stresses, BsrV racemase is expressed to release an impressive variety of high concentrations of NCDAA to the extracellular media. This wide production of NCDAA likely relies on different factors such as the broad range specificity of the racemase and the availability of the corresponding L-enantiomers. Nonetheless, the fact that Bsr enzymes are extraordinarily versatile (generate at least 10 DAA) suggests that this multispecific character is truly meaningful. In fact, a whole set of multispecific PG synthetic and modifying enzymes have coevolved with Bsr to permit PG incorporation and/or removal of NCDAA.

First, incorporation of NCDAA to the PG can be performed by distinct pathways. Although the Ldts-pathway for NCDAA incorporation is the only one that has been extensively investigated both in vitro and in vivo, it is likely that all alternative pathways display catalytic plasticity, of course depending on the organism. A possible coevolution of the multispecific activities governing production and incorporation is based on in vitro Ldt assays where the favored reactions are always those that generate modified muropeptides from canonical ones and not viceversa. ${ }^{18}$ This suggests that in the presence of NCDAA the PG will always tend to get edited, investment otherwise futile considering the enormous energetic expenditure that NCDAA production should cost for a cell population under nutrient starvation. The benefits of NCDAA incorporation will probably depend on the dosage of PG modification and thus, there would surely be additional layers of regulation. Supporting this, there is a notable number of publications in the $40 \mathrm{~s}$ and $60 \mathrm{~s}$ that showed the deleterious effect of high doses of exogenous DAA on diverse microbes. Given that Bsr are only expressed under stationary phase conditions, one regulation will come from the low levels of remaining L-AA in a spent media. Additionally, we speculate that Bsr and PG-editing enzymes might also be downregulated when certain levels of NCDAA incorporation have been reached.
Finally, it is clear that DD-carboxypeptidases are important enzymes to control the level of cross-linkage in the cell. ${ }^{66}$ However, given the novelty of NCDAA as PG modulators, it remains to be elucidated whether these activities can also control the levels of edited muropeptides. Production of NCDAA-pentapeptides is mediated by cytoplasmic PG-ligases and/or PBP-TP. However, the fact the NCDAA-pentapeptides are not detected in the PG of some organisms does not necessarily mean that they are not produced. One reason could be simply that there are DD-carboxypeptidases with distinct specificity on modified muropeptides. Indeed, this appears to be the case for $V$. cholerae and $E$. coli where canonical pentapeptides are never detected by HPLC. ${ }^{18}$ Nevertheless, $V$. cholerae PG from cultures supplemented with NCDAA displays detectable NCDAA-pentapeptides. These results underlie the inability of $V$. cholerae's DD-carboxypeptidases to cleave NCDAA-pentapeptides versus the efficient removal of the terminal D-Ala in the canonical ones. This substrate selectivity, as commented before for Ldts, favors the transition to a PG-edited state. By contrast, E. coli DD-carboxypeptidases are highly active on both canonical and NCDAAmuropeptides, despite their permissiveness with NCDAA incorporations at the fourth position of the peptide moieties.

Future studies will focus on investigating whether modifications on different muropeptide species represent different strategies of PG metabolism control. Moreover, it also remains to be seen whether the different selectivity exhibited by the NCDAA-incorporating and hydrolytic enzymes constitutes a defensive mechanism for non-producer organisms to withstand toxic NCDAA within poly-microbial communities. Finally, additional data revealing novel PGmodifying metabolites from the natural environment (i.e., as a result of interspecies communication or with the host) will interrogate the plasticity of PG-enzymes to modulate the architecture of the sacculus.

\section{Acknowledgments}

Research in the Cava Lab is supported by the Laboratory for Molecular Infection Medicine Sweden (MIMS), the Knut and Alice Wallenberg Foundation (KAW) and the Swedish Research Council. J.A.H. lab is supported by grants BFU2011-25326 (the Spanish Ministry of Economy and Competitiveness) and S2010/BMD-2457 (the Government of Community of Madrid).

\section{Disclosure Statement}

The authors declare no conflicts of interest.

\section{References}

1. Andersen, L.P., and L. Rasmussen. 2009. Helicobacter pylori-coccoid forms and biofilm formation. FEMS Immunol. Med. Microbiol. 56:112-115.

2. Anfora, A.T., B.J. Haugen, P. Roesch, P. Redford, and R.A. Welch. 2007. Roles of serine accumulation and catabolism in the colonization of the murine urinary tract by Escherichia coli CFT073. Infect. Immun. 75: 5298-5304.

3. Araki, Y., T. Nakatani, H. Hayashi, and E. Ito. 1971. Occurrence of non-N-substituted glucosamine residues in lysozyme-resistant peptidoglycan from Bacillus cereus cell walls. Biochem. Biophys. Res. Commun. 42:691-697. 
4. Babtie, A., N. Tokuriki, and F. Hollfelder. 2010. What makes an enzyme promiscuous? Curr. Opin. Chem. Biol. 14:200-207.

5. Barreteau, H., A. Kovac, A. Boniface, M. Sova, S. Gobec, and D. Blanot. 2008. Cytoplasmic steps of peptidoglycan biosynthesis. FEMS Microbiol. Rev. 32:168-207.

6. Bellais, S., M. Arthur, L. Dubost, J.E. Hugonnet, L. Gutmann, J. van Heijenoort, R. Legrand, J.P. Brouard, L. Rice, and J.L. Mainardi. 2006. Aslfm, the D-aspartate ligase responsible for the addition of D-aspartic acid onto the peptidoglycan precursor of Enterococcus faecium. J. Biol. Chem. 281:11586-11594.

7. Bera, A., R. Biswas, S. Herbert, E. Kulauzovic, C. Weidenmaier, A. Peschel, and F. Gotz. 2007. Influence of wall teichoic acid on lysozyme resistance in Staphylococcus aureus. J. Bacteriol. 189:280-283.

8. Biarrotte-Sorin, S., J.E. Hugonnet, V. Delfosse, J.L. Mainardi, L. Gutmann, M. Arthur, and C. Mayer. 2006. Crystal structure of a novel beta-lactam-insensitive peptidoglycan transpeptidase. J. Mol. Biol. 359:533-538.

9. Bielnicki, J., Y. Devedjiev, U. Derewenda, Z. Dauter, A. Joachimiak, and Z.S. Derewenda. 2006. B. subtilis ykuD protein at 2.0 A resolution: insights into the structure and function of a novel, ubiquitous family of bacterial enzymes. Proteins 62:144-151.

10. Boneca, I.G., O. Dussurget, D. Cabanes, M.A. Nahori, S. Sousa, M. Lecuit, E. Psylinakis, V. Bouriotis, J.P. Hugot, M. Giovannini, et al. 2007. A critical role for peptidoglycan $\mathrm{N}$-deacetylation in Listeria evasion from the host innate immune system. Proc. Natl. Acad. Sci. U. S. A. 104:997-1002.

11. Bopp, M. 1965. [Inhibition of Agrobacterium tumefaciens by D-amino acids]. Z. Naturforsch. B 20:899-905.

12. Bornscheuer, U.T., and R.J. Kazlauskas. 2004. Catalytic promiscuity in biocatalysis: using old enzymes to form new bonds and follow new pathways. Angew. Chem. Int. Ed. Engl. 43:6032-6040.

13. Both, D., E.M. Steiner, D. Stadler, Y. Lindqvist, R. Schnell, and G. Schneider. 2013. Structure of LdtMt2, an L,D-transpeptidase from Mycobacterium tuberculosis. Acta Crystallogr. D Biol. Crystallogr. 69:432-441.

14. Bouhss, A., A.E. Trunkfield, T.D. Bugg, and D. MenginLecreulx. 2008. The biosynthesis of peptidoglycan lipidlinked intermediates. FEMS Microbiol. Rev. 32:208-233.

15. Buynak, J.D. 2007. Cutting and stitching: the cross-linking of peptidoglycan in the assembly of the bacterial cell wall. ACS Chem. Biol. 2:602-605.

16. Cava, F., A. Hidalgo, and J. Berenguer. 2009. Thermus thermophilus as biological model. Extremophiles 13:213-231.

17. Cava, F., H. Lam, M.A. de Pedro, and M.K. Waldor. 2011. Emerging knowledge of regulatory roles of D-amino acids in bacteria. Cell Mol. Life Sci. 68:817-831.

18. Cava, F., M.A. de Pedro, H. Lam, B.M. Davis, and M.K. Waldor. 2011. Distinct pathways for modification of the bacterial cell wall by non-canonical D-amino acids. EMBO J. 30:3442-3453.

19. Conti, P., L. Tamborini, A. Pinto, A. Blondel, P. Minoprio, A. Mozzarelli, and C. De Micheli. 2011. Drug discovery targeting amino acid racemases. Chem. Rev. 111: 6919-6946.

20. Copley, S.D. 2003. Enzymes with extra talents: moonlighting functions and catalytic promiscuity. Curr. Opin. Chem. Biol. 7:265-272.

21. Correale, S., A. Ruggiero, R. Capparelli, E. Pedone, and R. Berisio. 2013. Structures of free and inhibited forms of the L,D-transpeptidase LdtMt1 from Mycobacterium tuberculosis. Acta Crystallogr. D Biol. Crystallogr. 69:16971706.

22. De Jonge, B.L., D. Gage, and N. Xu. 2002. The carboxyl terminus of peptidoglycan stem peptides is a determinant for methicillin resistance in Staphylococcus aureus. Antimicrob. Agents Chemother. 46:3151-3155.

23. Erdemli, S.B., R. Gupta, W.R. Bishai, G. Lamichhane, L.M. Amzel, and M.A. Bianchet. 2012. Targeting the cell wall of Mycobacterium tuberculosis: structure and mechanism of L,D-transpeptidase 2. Structure 20:2103-2115.

24. Espaillat, A., C. Carrasco-Lopez, N. Bernardo-Garcia, N. Pietrosemoli, L.H. Otero, L. Alvarez, M.A. de Pedro, F. Pazos, B.M. Davis, M.K. Waldor, et al. 2014. Structural basis for the broad specificity of a new family of amino-acid racemases. Acta Crystallogr. D Biol. Crystallogr. 70:79-90.

25. Fox, S., M. Fling, and N. Bollenback. 1944. Inhibition of bacterial growth by D-leucine. J. Biol. Chem. 155:465-468.

26. Gazi, A.D., S.N. Charova, N.J. Panopoulos, and M. Kokkinidis. 2009. Coiled-coils in type III secretion systems: structural flexibility, disorder and biological implications. Cell. Microbiol. 11:719-729.

27. Ghuysen, J.M. 1961. [Data on the structure of disaccharidepeptide complexes liberated from the wall of Micrococcus lysodeikticus by the action of beta(1-4)N-acetylhexosaminidases]. Biochim. Biophys. Acta 47:561-568.

28. Glasner, M.E., J.A. Gerlt, and P.C. Babbitt. 2006. Evolution of enzyme superfamilies. Curr. Opin. Chem. Biol. 10:492-497.

29. Glauner, B., J.V. Holtje, and U. Schwarz. 1988. The composition of the murein of Escherichia coli. J. Biol. Chem. 263:10088-10095.

30. Goodey, N.M., and S.J. Benkovic. 2008. Allosteric regulation and catalysis emerge via a common route. Nat. Chem. Biol. 4:474-482.

31. Grohs, P., L. Gutmann, R. Legrand, B. Schoot, and J.L. Mainardi. 2000. Vancomycin resistance is associated with serine-containing peptidoglycan in Enterococcus gallinarum. J. Bacteriol. 182:6228-6232.

32. Grula, E.A. 1960. Cell division in a species of Erwinia. I. Inhibition of division by D-amino acids. J. Bacteriol. 80: 375-385.

33. Hamada, S., M. Torii, S. Kotani, N. Masuda, T. Ooshima, K. Yokogawa, and S. Kawata. 1978. Lysis of Streptococcus mutans cells with mutanolysin, a lytic enzyme prepared from a culture liquor of Streptomyces globisporus 1829. Arch. Oral. Biol. 23:543-549.

34. Hammes-Schiffer, S., and S.J. Benkovic. 2006. Relating protein motion to catalysis. Annu. Rev. Biochem. 75:519-541.

35. Hancock, R. 1960. The amino acid composition of the protein and cell wall of Staphylococcus aureus. Biochim. Biophys. Acta 37:42-46.

36. Hash, J.H., and M.V. Rothlauf. 1967. The N,Odiacetylmuramidase of Chalaropsis species. I. Purification and crystallization. J. Biol. Chem. 242:5586-5590.

37. Henzler-Wildman, K., and D. Kern. 2007. Dynamic personalities of proteins. Nature 450:964-972.

38. Hermoso, J.A., L. Lagartera, A. Gonzalez, M. Stelter, P. Garcia, M. Martinez-Ripoll, J.L. Garcia, and M. Menendez. 2005. Insights into pneumococcal pathogenesis from the crystal structure of the modular teichoic acid phosphorylcholine esterase Pce. Nat. Struct. Mol. Biol. 12:533-538. 
39. Horcajo, P., M.A. de Pedro, and F. Cava. 2012. Peptidoglycan plasticity in bacteria: stress-induced peptidoglycan editing by noncanonical D-amino acids. Microb. Drug Resist. 18:306-313.

40. Inagaki, K., K. Tanizawa, H. Tanaka, and K. Soda. 1984. Purification and properties of amino acid racemase from Aeromonas punctata subsp. caviae. Prog. Clin. Biol. Res. 144A:355-363.

41. Kamerlin, S.C., and A. Warshel. 2010. At the dawn of the 21st century: is dynamics the missing link for understanding enzyme catalysis? Proteins 78:1339-1375.

42. Kazlauskas, R.J. 2005. Enhancing catalytic promiscuity for biocatalysis. Curr. Opin. Chem. Biol. 9:195-201.

43. Kim, H.S., J. Kim, H.N. Im, J.Y. Yoon, D.R. An, H.J. Yoon, J.Y. Kim, H.K. Min, S.J. Kim, J.Y. Lee, et al. 2013. Structural basis for the inhibition of Mycobacterium tuberculosis L,D-transpeptidase by meropenem, a drug effective against extensively drug-resistant strains. Acta Crystallogr. D Biol. Crystallogr. 69:420-431.

44. Kobayashi, Y., M. Fling, and S.W. Fox. 1948. Antipodal specificity in the inhibition of growth of Escherichia coli by amino acids. J. Biol. Chem. 174:391-398.

45. Kokkinidis, M., N.M. Glykos, and V.E. Fadouloglou. 2012. Protein flexibility and enzymatic catalysis. Adv. Protein Chem. Struct. Biol. 87:181-218.

46. Kolodkin-Gal, I., D. Romero, S. Cao, J. Clardy, R. Kolter, and R. Losick. 2010. D-amino acids trigger biofilm disassembly. Science 328:627-629.

47. Kuru, E., H.V. Hughes, P.J. Brown, E. Hall, S. Tekkam, F. Cava, M.A. de Pedro, Y.V. Brun, and M.S. Vannieuwenhze. 2012. In situ probing of newly synthesized peptidoglycan in live bacteria with fluorescent D-amino acids. Angew. Chem. Int. Ed. Engl. 51:12519-12523.

48. Lam, H., D.C. Oh, F. Cava, C.N. Takacs, J. Clardy, M.A. de Pedro, and M.K. Waldor. 2009. D-amino acids govern stationary phase cell wall remodeling in bacteria. Science 325:1552-1555.

49. Langmead, C.J. 2014. Generative models of conformational dynamics. Adv. Exp. Med. Biol. 805:87-105.

50. Lark, C., and K.G. Lark. 1959. The effects of D-amino acids on Alcaligenes fecalis. Can. J. Microbiol. 5:369-379.

51. Lebar, M.D., T.J. Lupoli, H. Tsukamoto, J.M. May, S. Walker, and D. Kahne. 2013. Forming cross-linked peptidoglycan from synthetic gram-negative Lipid II. J. Am. Chem. Soc. 135:4632-4635.

52. Leguina, J.I., J.C. Quintela, and M.A. de Pedro. 1994. Substrate specificity of Escherichia coli LD-carboxypeptidase on biosynthetically modified muropeptides. FEBS Lett. 339:249-252.

53. Leiman, S.A., J.M. May, M.D. Lebar, D. Kahne, R. Kolter, and R. Losick. 2013. D-amino acids indirectly inhibit biofilm formation in Bacillus subtilis by interfering with protein synthesis. J. Bacteriol. 195:5391-5395.

54. Li, C., and M. Liu. 2013. Protein dynamics in living cells studied by in-cell NMR spectroscopy. FEBS Lett. 587: 1008-1011.

55. Liechti, G.W., E. Kuru, E. Hall, A. Kalinda, Y.V. Brun, M. Vannieuwenhze, and A.T. Maurelli. 2013. A new metabolic cell-wall labelling method reveals peptidoglycan in Chlamydia trachomatis. Nature 506:507-510.

56. Lim, Y.H., K. Yokoigawa, N. Esaki, and K. Soda. 1993. A new amino acid racemase with threonine alpha-epimerase activity from Pseudomonas putida: purification and characterization. J. Bacteriol. 175:4213-4217.
57. Magnet, S., L. Dubost, A. Marie, M. Arthur, and L. Gutmann. 2008. Identification of the L,D-transpeptidases for peptidoglycan cross-linking in Escherichia coli. J. Bacteriol. 190:4782-4785.

58. Magnet, S., S. Bellais, L. Dubost, M. Fourgeaud, J.L. Mainardi, S. Petit-Frere, A. Marie, D. MenginLecreulx, M. Arthur, and L. Gutmann. 2007. Identification of the L,D-transpeptidases responsible for attachment of the Braun lipoprotein to Escherichia coli peptidoglycan. J. Bacteriol. 189:3927-3931.

59. Mainardi, J.L., M. Fourgeaud, J.E. Hugonnet, L. Dubost, J.P. Brouard, J. Ouazzani, L.B. Rice, L. Gutmann, and M. Arthur. 2005. A novel peptidoglycan cross-linking enzyme for a beta-lactam-resistant transpeptidation pathway. J. Biol. Chem. 280:38146-38152.

60. Matsumura, I., and A.D. Ellington. 2001. In vitro evolution of beta-glucuronidase into a beta-galactosidase proceeds through non-specific intermediates. J. Mol. Biol. 305: 331-339.

61. Nashine, V.C., S. Hammes-Schiffer, and S.J. Benkovic. 2010. Coupled motions in enzyme catalysis. Curr. Opin. Chem. Biol. 14:644-651.

62. O'Connor, K.A., and D.R. Zusman. 1997. Starvationindependent sporulation in Myxococcus xanthus involves the pathway for beta-lactamase induction and provides a mechanism for competitive cell survival. Mol. Microbiol. 24:839-850.

63. Ohno, N., T. Yadomae, and T. Miyazaki. 1982. Identification of 2-amino-2-deoxyglucose residues in the peptidoglucan of Streptococcus pneumoniae. Carbohydr. Res. 107:152-155.

64. Otero, L.H., A. Rojas-Altuve, L.I. Llarrull, C. CarrascoLopez, M. Kumarasiri, E. Lastochkin, J. Fishovitz, M. Dawley, D. Hesek, M. Lee, et al. 2013. How allosteric control of Staphylococcus aureus penicillin binding protein 2a enables methicillin resistance and physiological function. Proc. Natl. Acad. Sci. U. S. A. 110:16808-16813.

65. Park, J.T., and J.L. Strominger. 1957. Mode of action of penicillin. Science 125:99-101.

66. Park, J.T., and T. Uehara. 2008. How bacteria consume their own exoskeletons (turnover and recycling of cell wall peptidoglycan). Microbiol. Mol. Biol. Rev. 72:211-227.

67. Reynolds, P.E., and P. Courvalin. 2005. Vancomycin resistance in enterococci due to synthesis of precursors terminating in D-alanyl-D-serine. Antimicrob. Agents Chemother. 49:21-25.

68. Rogers, H.J., H.R. Perkins, and J.B. Ward. 1980. Microbial Cell Walls and Membranes. London: Chapman \& Hall.

69. Roise, D., K. Soda, T. Yagi, and C.T. Walsh. 1984. Inactivation of the Pseudomonas striata broad specificity amino acid racemase by $\mathrm{D}$ and $\mathrm{L}$ isomers of beta-substituted alanines: kinetics, stoichiometry, active site peptide, and mechanistic studies. Biochemistry 23:5195-5201.

70. Rousset, A., M. Nguyen-Disteche, R. Minck, and J.M. Ghuysen. 1982. Penicillin-binding proteins and carboxypeptidase/transpeptidase activities in Proteus vulgaris P18 and its penicillin-induced stable L-forms. J. Bacteriol. 152: 1042-1048.

71. Scheffers, D.J., and M.G. Pinho. 2005. Bacterial cell wall synthesis: new insights from localization studies. Microbiol. Mol. Biol. Rev. 69:585-607.

72. Siegrist, M.S., S. Whiteside, J.C. Jewett, A. Aditham, F. Cava, and C.R. Bertozzi. 2013. D-amino acid chemical 
reporters reveal peptidoglycan dynamics of an intracellular pathogen. ACS Chem. Biol. 8:500-505.

73. Sieradzki, K., and A. Tomasz. 1996. A highly vancomycin-resistant laboratory mutant of Staphylococcus aureus. FEMS Microbiol. Lett. 142:161-166.

74. Teeri, A.E. 1954. Effect of D-amino acids on growth of lactobacilli. J. Bacteriol. 67:686-688.

75. Teilum, K., J.G. Olsen, and B.B. Kragelund. 2011. Protein stability, flexibility and function. Biochim. Biophys. Acta 1814:969-976.

76. Tuttle, A.L., and H. Gest. 1960. Induction of morphological aberrations in Rhodospirillum rubrum by D-amino acids. J. Bacteriol. 79:213-216.

77. Typas, A., M. Banzhaf, C.A. Gross, and W. Vollmer. 2011. From the regulation of peptidoglycan synthesis to bacterial growth and morphology. Nat. Rev. Microbiol. 10:123-136.

78. van der Donk, W.A. 2006. Lighting up the nascent cell wall. ACS Chem. Biol. 1:425-428.

79. van Heijenoort, J. 2007. Lipid intermediates in the biosynthesis of bacterial peptidoglycan. Microbiol. Mol. Biol. Rev. 71:620-635.

80. Veiga, P., S. Piquet, A. Maisons, S. Furlan, P. Courtin, M.P. Chapot-Chartier, and S. Kulakauskas. 2006. Identification of an essential gene responsible for D-Asp incorporation in the Lactococcus lactis peptidoglycan crossbridge. Mol. Microbiol. 62:1713-1724.

81. Vollmer, W. 2008. Structural variation in the glycan strands of bacterial peptidoglycan. FEMS Microbiol. Rev. 32:287-306.

82. Vollmer, W., and A. Tomasz. 2000. The pgdA gene encodes for a peptidoglycan $\mathrm{N}$-acetylglucosamine deacetylase in Streptococcus pneumoniae. J. Biol. Chem. 275:2049620501.

83. Vollmer, W., and U. Bertsche. 2008. Murein (peptidoglycan) structure, architecture and biosynthesis in Escherichia coli. Biochim. Biophys. Acta 1778:1714-1734.

84. Vollmer, W., D. Blanot, and M.A. de Pedro. 2008. Peptidoglycan structure and architecture. FEMS Microbiol. Rev. 32:149-167.
85. Watanabe, A., T. Yoshimura, B. Mikami, H. Hayashi, H. Kagamiyama, and N. Esaki. 2002. Reaction mechanism of alanine racemase from Bacillus stearothermophilus: $\mathrm{x}-$ ray crystallographic studies of the enzyme bound with N-(5'-phosphopyridoxyl)alanine. J. Biol. Chem. 277:1916619172.

86. Weidel, W., and H. Pelzer. 1964. Bagshaped macromolecules-a new outlook on bacterial cell walls. Adv. Enzymol. Relat. Areas Mol. Biol. 26:193-232.

87. Wood, W.A., and I.C. Gunsalus. 1951. D-Alanine formation; a racemase in Streptococcus faecalis. J. Biol. Chem. 190:403-416.

88. Yaw, K.E., and J.C. Kakavas. 1952. Studies on the effects of D-amino acids on Brucella abortus. J. Bacteriol. 63: 263-268.

89. Yorifuji, T., H. Misono, and K. Soda. 1971. Arginine racemase of Pseudomonas-graveolens. 2. Racemization and transamination of ornithine catalyzed by arginine racemase. J. Biol. Chem. 246:5093-5101.

90. Yorifuji, T., K. Ogata, and K. Soda. 1971. Arginine racemase of Pseudomonas graveolens. 1. Purification, crystallization, and properties. J. Biol. Chem. 246:5085-5092.

91. Yoshimura, T., and N. Esak. 2003. Amino acid racemases: functions and mechanisms. J. Biosci. Bioeng. 96: 103-109.

92. Zipperle, G.F., Jr., J.W. Ezzell, Jr., and R.J. Doyle. 1984. Glucosamine substitution and muramidase susceptibility in Bacillus anthracis. Can. J. Microbiol. 30:553-559.

Address correspondence to:

Felipe Cava, PhD

Laboratory for Molecular Infection Medicine Sweden

Department of Molecular Biology

Umea Centre for Microbial Research

Umea University

Umea SE-901 87

Sweden

E-mail: felipe.cava@molbiol.umu.se 